\title{
EDUCACIÓN ESCOLAR Y EDUCACIÓN SUPERIOR. UN DIÁLOGO NECESARIO PARA CONSTRUIR RELACIONES SISTÉMICAS
}

\section{El perfil de ingreso a la educación superior y el perfil de egreso de la educación escolar}

Dentro de la educación superior, principalmente universitaria, se suele afirmar que un porcentaje relevante de los estudiantes que accede a primer año no domina algunos contenidos considerados fundamentales para desarrollar con éxito los programas de estudio de los cursos iniciales, lo que ocurriría por una de las siguientes razones, o por una combinación de ambas:

- La educación escolar no estaría logrando lo que declara en sus definiciones curriculares, fundamentalmente en la enseñanza media.

- La educación superior estaría exigiendo el dominio de algunos contenidos que no se encuentran presentes en los aprendizajes del nivel anterior.

Siendo éste el principal punto de roce entre ambos mundos educacionales, resulta necesario partir analizando la validez de estas dos posibles explicaciones.

\section{Los resultados del aprendizaje escolar}

La Encuesta de Alfabetización de la Población Adulta (Bravo, 2001) muestra que en Chile más de la mitad de la población mayor de 15 años no entiende lo que lee y sólo es capaz de realizar inferencias muy básicas utilizando material impreso, situación que aumenta con la edad y en la medida en que se tiene menos escolaridad. 
Estos datos están señalando que el manejo deficitario de habilidades básicas se arrastra desde hace tiempo y que afecta con más fuerza a quienes se desenvuelven en un contexto de marginalidad. Desde otra perspectiva, la mejor posición que se detecta en los más jóvenes está indicando que los esfuerzos desplegados en las últimas décadas para aumentar la cobertura y renovar la enseñanza escolar están entregando algunos frutos.

Por otro lado, las pruebas nacionales, construidas a partir de los contenidos establecidos en el currículo escolar, como las que aplica el Sistema de Medición de la Calidad de la Educación (SIMCE) o la Prueba de Selección Universitaria (PSU), muestran que los resultados del aprendizaje se ordenan según la jerarquía socioeconómica de los estudiantes, desigualdad que se acrecienta en la educación media (García Huidobro y Bellei, 2003).

En otra vertiente, el programa Liceo para Todos ${ }^{1}$ ha detectado que al iniciar el primer año medio casi la mitad de los estudiantes presenta desempeños en comprensión y expresión oral y escrita que corresponden al $5^{\circ}$ año básico, mientras dos terceras partes se encuentran en un nivel similar en Matemáticas, pudiendo resolver problemas sólo con las cuatro operaciones en números naturales, con gran dificultad cuando incluye operatorias combinadas (MINEDUC, 2005).

De acuerdo con estas constataciones, resulta evidente que no todos los estudiantes alcanzan los aprendizajes que el currículo define como deseables para la experiencia escolar, siendo los más afectados aquellos niños y jóvenes provenientes de grupos sociales que, hasta hace poco, se mantenían fuera de las aulas escolares, que están accediendo masivamente a ellas, y que, además, deben internalizar los contenidos cada vez más complejos que demanda la sociedad del conocimiento.

1 Iniciativa del Ministerio de Educación de Chile orientada a disminuir la deserción escolar en aquellos establecimientos de educación media que atienden la población de mayor vulnerabilidad social y educativa. 


\section{Las demandas de la educación superior}

La segunda explicación (la educación superior exigiría el dominio de aprendizajes no contemplados en las definiciones curriculares de la educación escolar) resulta difícil de comprobar, al no contar con estudios que permitan comparar el perfil de egreso de la enseñanza media con los perfiles de ingreso a las diferentes carreras de educación superior ${ }^{2}$. Sin embargo, el análisis de las transformaciones que ha venido experimentando la escuela secundaria, a partir de mediados del siglo pasado, hace posible suponer que esta explicación podría tener su fundamento. Esta institución, nacida en íntima vinculación con la universidad, ha ido abandonando su carácter exclusivo de institución preuniversitaria para enfatizar la preparación de personas que puedan integrarse creativamente en la sociedad como ciudadanos y agentes económicos, y que deban desenvolverse en un medio que exige el aprendizaje a lo largo de la vida. Formar para la vida y para aprender a aprender resulta distinto a preparar para un buen desempeño cuando se ingrese a la educación superior.

Ese fue el sentido con el cual se estructuró la reforma educativa de fines de los años sesenta en el país, privilegiando su conexión con la escuela básica ${ }^{3}$, donde, además, se integró la preparación técnico-profesional como una alternativa válida de educación media junto con la tradicional de formación preuniversitaria. Por lo tanto, el currículo de este nivel educativo deja de construirse para garantizar el buen desempeño de la minoría que va a acceder de manera inmediata a la educación superior, aunque mantiene, dentro de sus espacios de diversificación vocacional, alternativas que lo hagan posible.

2 Comparación que se dificulta al considerar la diversidad de definiciones curriculares presentes en la educación superior, a diferencia de la educación escolar donde existe un marco curricular que establece los objetivos fundamentales y contenidos mínimos para todo el sistema.

3 Conexión que culmina con la reforma que establece 12 años como escolaridad obligatoria, haciendo de los niveles de educación básica y media un espacio con continuidad. 
Por otro lado, los diseños curriculares más recientes en la educación secundaria han abandonado el postulado de que en este ciclo se debe cerrar la formación general, posibilitando que la educación superior se dedique a la preparación especializada, ya que cada día se suman evidencias que indican que las habilidades básicas se siguen desarrollando en niveles de mayor complejidad, en la medida en que se progresa en el aprendizaje. Ejemplo de esto es lo que han venido haciendo universidades en países desarrollados en el campo de la "escritura académica", donde se parte del supuesto de que se trata de una habilidad que sólo se puede lograr en este nivel educativo, formando la capacidad de argumentar, organizar ideas y razonamientos y comunicar ideas con claridad y precisión (Carlino, s/f). Es decir, lo que se espera en la enseñanza primaria o secundaria es que se utilice una escritura apropiada a las necesidades expresivas propias de esos niveles, entendiendo que en la educación superior se requiere progresar en su dominio.

Probablemente, esta idea se encuentra en la base de aquellos bachilleratos universitarios que se orientan a entregar oportunidades para progresar en el manejo de habilidades de aplicación general, requeridas para un estudio de nivel superior ligadas a las necesidades de desempeño profesional, como pueden ser trabajar con personas diversas, comunicarse de forma asertiva y efectiva o fundamentar la toma de decisiones.

\section{Enfrentando los efectos y las causas}

Partiendo de la convicción de que las deficiencias observadas en los aprendizajes de los estudiantes que ingresan a la educación superior no se resolverán en un plazo breve, en muchas instituciones de educación superior se han implementado procesos de nivelación que, en algunos casos, abarcan a la totalidad de los estudiantes y, en otros, a aquellos que han demostrado un desempeño deficiente en pruebas de diagnóstico. 
Se trata de una medida remedial eficaz que enfrenta los efectos visibles del problema inmediato y, por lo tanto, necesaria. Sin embargo, para resolverlo se necesita actuar sobre sus causas y con visión de largo plazo.

De acuerdo con la evaluación efectuada por la Organización para la Cooperación y el Desarrollo Económicos (OECD, en su sigla en inglés), las políticas curriculares implementadas recientemente en el país se orientan en la dirección correcta y se puede esperar que rindan frutos si se enfrenta la "brecha de capacidad" en la fuerza docente, provocada por una inadecuada preparación para enseñar de acuerdo con los niveles de práctica relativamente altos requeridos por la reforma (OECD, 2004, p. 291).

Los estudios de cobertura curricular efectuados por la Unidad de Currículum y Evaluación del Ministerio de Educación muestran que la mayoría de los profesores no llega a tratar más allá del $80 \%$ de los objetivos y contenidos que debería abarcar en un año escolar. En la encuesta aplicada en TIMSS 1999 a los profesores y profesoras de Matemáticas y Ciencias de $8^{\circ}$ año básico, éstos se manifestaron inseguros de su dominio de la asignatura.

Algunos diagnósticos indican que éstas y otras carencias del quehacer docente se fundamentan principalmente en las características que han venido asumiendo la formación inicial y el perfeccionamiento continuo, por encima de las condiciones de trabajo y salariales que han experimentado un mejoramiento sostenido en el último tiempo.

En la preparación y actualización de los profesores no se logra un balance adecuado entre los contenidos propios del ámbito disciplinario y los vinculados con la práctica pedagógica y evaluativa. Respecto de esta última, se observa una tendencia a centrarla en contenidos muy generales desasociados del perfil de competencias laborales propio de la docencia, el cual enfatiza la capacidad para gestionar, de manera efectiva, ambientes, situaciones 
y recursos que faciliten el acceso al aprendizaje a personas diversas en contextos diferentes.

Y como en el país tanto la formación inicial como la capacitación continua de los profesores es realizada por instituciones de educación superior ${ }^{4}$, éstas cuentan aquí con una oportunidad privilegiada para enfrentar esa "brecha de capacidad" detectada por la OECD que está influyendo decisivamente en los resultados de la educación escolar.

\section{Acercando posiciones}

Chile necesita dar un salto cualitativo en educación para alcanzar las expectativas de desarrollo y de crecimiento económico que se han venido planteando en el último tiempo. Las pruebas internacionales aplicadas en el país ${ }^{5}$ demuestran que los puntajes obtenidos por los estudiantes chilenos son bajos en relación con los que alcanzan nuestros socios en pactos y tratados de libre comercio y, sobre todo, en la perspectiva de lograr los aprendizajes que son indispensables en el mundo contemporáneo (MINEDUC, 2003 y MINEDUC, 2004), incluyendo a quienes asisten a escuelas privadas pagadas (OECD, 2004, p. 174).

La crítica de la educación superior hacia los resultados de la educación escolar no presenta novedad, ya que se suele emitir con cierta frecuencia hacia el nivel inferior de enseñanza cuando se encuentran desarticulados los espacios formativos que deberían constituir un sistema. En los establecimientos escolares que matriculan estudiantes el primer año de educación media los profesores tienden a quejarse de la preparación con que llegan desde la educación básica, lo que no ocurre donde hay continuidad de estudios entre estos niveles.

4 Incluyendo los denominados programas especiales de regularización de título para profesores, donde se propone una preparación acelerada de escasa profundidad y baja exigencia.

5 Principalmente TIMSS 1999 y 2003 (Trends in International Mathematics and Science Study) y PISA 2000 (Programme for Internacional Student Assessment). 
Por lo tanto, para poder dar ese necesario salto cualitativo, un factor clave consiste en articular los componentes del sistema educativo, estableciendo una alianza donde cada nivel aporte al propósito compartido de ofrecer oportunidades de progresar en el aprendizaje a lo largo de la vida.

La Conferencia Regional sobre Políticas y Estrategias para la Transformación de la Educación Superior en América Latina y el Caribe, convocada por la UNESCO en La Habana, en noviembre de 1996, dejó planteada esta perspectiva, al sostener la necesidad de "integrar las instituciones de educación superior con los demás niveles de la educación, con el objeto de conformar un sistema articulado dentro del cual deberán asumir una conducta proactiva conducente a la identificación de falencias. Su contribución podrá traducirse a través de la investigación aplicada, de su participación en la formación inicial de docentes, y en el perfeccionamiento y actualización del personal calificado, y el asesoramiento en el diseño de modelos curriculares".

En el país hay iniciativas que muestran la factibilidad de vincularse, así como los beneficios que trae la asociación. Ejemplo de ello son:

- La Mesa de Trabajo sobre Nuevas Pruebas de Selección Universitaria (2002), que elaboró el referente curricular de éstas y concordó los criterios para excluir contenidos curriculares en el periodo de transición hasta 2006.

- El Programa Educacional para Niños con Talentos Académicos (PENTA UC), iniciado en la Pontificia Universidad Católica de Chile y extendido a otras universidades regionales, que ofrece servicios educacionales complementarios a la experiencia escolar a estudiantes de educación media y de los últimos cursos de educación básica en las áreas de Ciencias Sociales, Ciencias Naturales, Humanidades y Matemáticas.

- Los proyectos de articulación de la formación técnica impulsados por el Programa Chilecalifica, donde se están asociando instituciones educativas de nivel medio y superior 
con organizaciones productivas para mejorar la oferta de formación técnica en un sector económico, articulándola en una perspectiva de formación permanente.

Esta última experiencia está empezando a resolver el desajuste entre los perfiles de egreso de la educación media técnica y los de ingreso de la educación técnica de nivel superior. También está entregando pistas para hacerse cargo de la diversidad de formaciones con la cual acceden los estudiantes a la formación técnica, incluyendo a quiénes lo hacen desde la modalidad humanístico-científica sin preparación previa. Por último, intenta generar procedimientos para reconocer lo aprendido en la capacitación en servicio o por medios informales ${ }^{6}$. Por lo tanto, estos proyectos deberían analizarse como un modelo que podría extenderse a otras áreas de desempeño profesional.

Un ámbito privilegiado de vinculación debería ser la práctica profesional que realizan los estudiantes de pedagogía en establecimientos escolares, inscribiéndolas en proyectos de investigación y de acción orientados a promover el mejoramiento de la calidad y la equidad de la educación, beneficiándose dichos establecimientos de esta asistencia técnica y las escuelas formadoras de maestros de un contraste permanente de sus propuestas formativas con las exigencias de desempeño del perfil de competencias del profesor.

Acercar posiciones es la herramienta más poderosa para enfrentar desafíos complejos de gran envergadura. Así lo demuestra el consenso alcanzado en la Comisión Nacional para la Modernización de la Educación (1994) para definir una carta de navegación orientadora de las transformaciones que ha venido experimentando la educación escolar. También, la concertación alcanzada entre el medio educativo y el mundo productivo para realizar una transformación profunda de la educación técnica

6 Iniciativa clave en una sociedad que valora el dominio del conocimiento sin importar el espacio o la fuente donde haya sido adquirido. 
en el país, lo que ha supuesto poner a trabajar a dos culturas organizacionales que se necesitaban pero que estaban alejadas por mutuas sospechas (Miranda, 2003).

Para resolver el problema que hemos venido describiendo y entregar oportunidades de formación que atiendan, con propuestas de calidad, a la creciente diversidad de personas que está accediendo a todos los niveles educativos, parece necesario generar espacios donde los actores de la educación escolar y de la educación superior se sienten en una misma mesa, dando a conocer cuáles son sus trayectorias de desarrollo, hasta descubrir aquellos puntos del camino donde se pueden encontrar. Allí la educación superior podría conocer y discutir las definiciones curriculares que existen en la educación media, cuánto se aborda y cuánto se logra de ellas. Por su parte, la educación escolar podría conocer y analizar las demandas que surgen del medio académico, abriendo una instancia para negociarlas. $\mathrm{Y}$, entre ambos, construir un lugar orientado al desarrollo de las capacidades requeridas para incrementar la calidad de la educación escolar en aquel ámbito de la educación superior ocupado del desarrollo de la pedagogía.

\section{Referencias bibliográficas}

Carlino, Paula. Enseñar a escribir en la universidad: cómo lo hacen en Estados Unidos y por qué. En: Revista Iberoamericana de Educación, Madrid, OEI.

Comisión de las Comunidades Europeas. Memorándum sobre el aprendizaje permanente. Documento de trabajo, Bruselas, 2000.

Comisión Nacional para la Modernización de la Educación. Los desafíos de la educación chilena frente al siglo XXI, Editorial Universitaria, Santiago de Chile, 1994.

Bravo, David y Contreras, Dante. Competencias Básicas de la Población Adulta. CORFO/Universidad de Chile/Ministerio de Economía, Fomento y Reconstrucción, Santiago de Chile, 2001. 
García-Huidobro, Juan Eduardo y Belleï, Cristian. Desigualdad Educativa en Chile. En: La Educación en Chile, Hoy. Ediciones Universidad Diego Portales, Santiago de Chile, 2003.

MINEDUC. Decreto Supremo No 220. Objetivos Fundamentales y Contenidos Mínimos Obligatorios para la Enseñanza Media. Ministerio de Educación, Santiago de Chile, 1998.

MINEDUC. Chile y el aprendizaje de matemáticas y ciencias según TIMSS. Resultados de los estudiantes chilenos de $8^{\circ}$ básico en el Estudio Internacional de Tendencias en Matemáticas y Ciencias. Ministerio de Educación, Santiago de Chile, 2003.

MINEDUC. Competencias para la vida. Resultados de los estudiantes chilenos en el estudio PISA 2000. Ministerio de Educación, Santiago de Chile, 2004.

MINEDUC. Nivelación Restitutiva en Liceos para Todos; Innovación para la Calidad. Ministerio de Educación, Documento de Trabajo, Santiago de Chile, 2005.

Miranda, Martín. Transformación de la Educación Media Técnicoprofesional. En: Políticas Educacionales en el cambio de siglo. La reforma del sistema escolar chileno. Editorial Universitaria, Santiago de Chile, 2003.

The World Bank. Expanding Opportunities and Building Competencies for Young People. A New Agenda for Secondary Education. Washington, DC.

OECD. Revisión de Políticas Nacionales: Chile. París, 2004.

UNESCO. Conferencia Regional sobre Políticas y Estrategias para la Transformación de la Educación Superior en América Latina y el Caribe. La Habana, Cuba, 1996. 